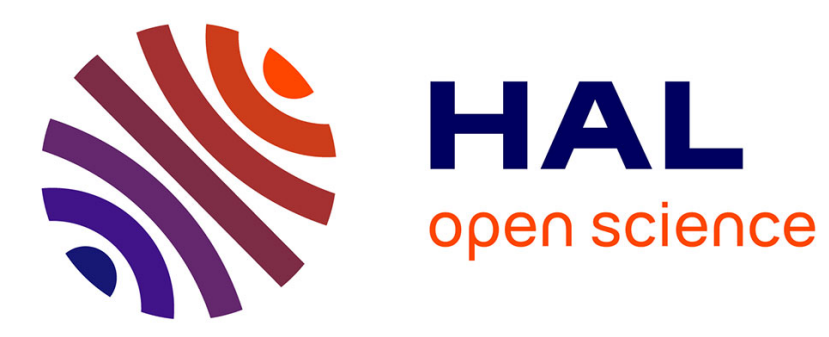

\title{
Fault detection In electrical drives - approach signal
}

\author{
Abdelghani Chahmi, Mokhtar Bendjebbar, Bertrand Raison
}

\section{To cite this version:}

Abdelghani Chahmi, Mokhtar Bendjebbar, Bertrand Raison. Fault detection In electrical drives approach signal. 2014 International Conference on Electrical Sciences and Technologies in Maghreb (CISTEM 2014) , Nov 2014, tunis, Tunisia. pp.1 - 6, 10.1109/CISTEM.2014.7076938 . hal-01376761

\section{HAL Id: hal-01376761 \\ https://hal.science/hal-01376761}

Submitted on 6 Oct 2016

HAL is a multi-disciplinary open access archive for the deposit and dissemination of scientific research documents, whether they are published or not. The documents may come from teaching and research institutions in France or abroad, or from public or private research centers.
L'archive ouverte pluridisciplinaire HAL, est destinée au dépôt et à la diffusion de documents scientifiques de niveau recherche, publiés ou non, émanant des établissements d'enseignement et de recherche français ou étrangers, des laboratoires publics ou privés. 


\section{Détection De Défaillances Sur Un Entraînement Electrique-Approche Signal-}

\author{
Abdelghani CHAHMI,Mokhtar BENDJEBBAR \\ Laboratoire développement des entraînements électriques \\ Université des Sciences et Technologie \\ Oran, Algérie \\ Abdelghani.chahmi@gmail.com, Bendjebb_dz@yahoo.fr
}

\author{
Bertrand RAISON \\ Laboratoire de Génie Electrique de Grenoble G2ELab \\ Institut National de Polytechnique \\ Grenoble, France \\ Bertrand. Raison@g2elab.grenoble-inp.fr
}

\begin{abstract}
RESUME - Cet article traite de la détection de défauts dans les entrainements électriques et en particulier ceux à base de machine asynchrone. D'une part, on démontre la caractérisation des processus de défaillance et l'obtention de modèles de ces dysfonctionnements. D'autre part le développement de diagnostic de la machine. Les études de dysfonctionnement dans les entrainements ne pouvant faire appel qu'à peu de données expérimentales, il a été indispensable de nous munir d'outils de simulation qui ont servi à caractériser les comportements défaillants. La détection de défauts fait appel aux techniques de traitement du signal dans les phases de fonctionnement connues (vitesse fixe). Finalement, nous nous intéressons a développé l'algorithme de modélisation de la machine asynchrone saine et défaillant par la signature fréquentielle (apparition des raies, amplitudes de la composante continue,......).
\end{abstract}

MOT-CLES- Moteur asynchrone, Modélisation, Défaut rotorique, Défaut statorique, Analyse fréquentielle.

\section{INTRODUCTION}

Les entrainements électriques à base de machine asynchrone sont largement utilisés dans les applications industrielles en raison de leur faible cout, de leurs performances et de leur robustesse. Les systèmes industriels devenant de plus en plus complexes et sophistiqués, il est nécessaire de pouvoir garantir la sûreté de leur fonctionnement. On entend par sûreté de fonctionnement, la capacité qu'aura un système à effectuer les tâches pour lesquelles il a été conçu. Cette capacité peut être entravée par les défauts affectant le fonctionnement du système, entraînant ainsi des conséquences inacceptables sur la sécurité (des hommes ou du matériel), l'environnement et la qualité de production ou de service. Il est essentiel de mettre en place des fonctions de surveillance permettant de garantir le fonctionnement sûr des systèmes. On entend par fonctions de surveillance l'ensemble des actions permettant de détecter, localiser et diagnostiquer tout phénomène anormal survenant pendant le fonctionnement. Ces fonctions de surveillance sont élaborées à partir des techniques dites DLD (Détection et localisation de Défauts) ou FDI (Fault Détection and Isolation).Les références [1]-[2] passent en revue un grand nombre de cas de défaillances, ainsi que des techniques de détection associées. La raison principale des défaillances de machine reste les défauts de roulement à bille
[4]-[5] qui ont de nombreuses causes telles que la contamination du lubrifiant, une charge excessive ou encore des causes électriques. Dans cet article, un récapitulatif des modèles des défauts, choix de la variable à étudier, de l'influence des boucles de contrôle et les signatures spectrales du courant statorique est donné dans le paragraphe II. Le paragraphe III rappelle quant à lui les effets mécaniques des défauts de roulements et la formulation d'oscillation du couple de charge.

\section{LES DÉFAILLANCES ÉLECTRIQUES}

\section{A. Les modeles des défauts dans la machine}

Il faut noter que cette modélisation est critiquable: les défauts de type court-circuit sont représenté dans le modèle comme des suppressions de spires c'est-à-dire des ouvertures partielles de phase ; cela ne correspond pas tout à fait à la réalité puisqu'il faudrait aussi tenir compte des spires en courtcircuit. Ce modèle constitue un progrès par rapport aux modèles équilibrés ou /et ne considérant qu'une variation de résistance couramment utilisés pour valider les algorithmes. Sous les conditions rappelées au paragraphe précèdent, les équations des circuits électriques font intervenir des inductances propres et mutuelles permettant de définir les flux en fonction des courants [9]. Au niveau du stator, les tensions vérifient :

$$
\left[\begin{array}{l}
v_{s a} \\
v_{s b} \\
v_{s c}
\end{array}\right]=\left[R_{s}\right]\left[\begin{array}{l}
i_{s a} \\
i_{s b} \\
i_{s c}
\end{array}\right]+\frac{d}{d t}\left[\begin{array}{l}
\varphi_{s a} \\
\varphi_{s b} \\
\varphi_{s c}
\end{array}\right]
$$

Ou la matrice des résistances s'écrit d'après l'hypothèse de symétrie :

$$
\left[R_{s}\right]=\left[\begin{array}{ccc}
r_{s} & 0 & 0 \\
0 & r_{s} & 0 \\
0 & 0 & r_{s}
\end{array}\right]
$$

$\mathrm{Au}$ niveau du rotor, sous l'hypothèse que le rotor est assimilable à un rotor avec enroulement triphasés, les tensions s'écrivent : 


$$
\left[\begin{array}{l}
v_{r a} \\
v_{r b} \\
v_{r c}
\end{array}\right]=\left[R_{R}\right]\left[\begin{array}{l}
i_{r a} \\
i_{r b} \\
i_{r c}
\end{array}\right]+\frac{\mathrm{d}}{\mathrm{dt}}\left[\begin{array}{l}
\varphi_{r a} \\
\varphi_{r b} \\
\varphi_{r c}
\end{array}\right]
$$

La matrice $R_{R}$ posséde la méme forme que $R_{S}$

Les flux s'expriment alors grâce aux matrices inductances et aux courants

$$
\begin{gathered}
{\left[\begin{array}{l}
\varphi_{s a} \\
\varphi_{s b} \\
\varphi_{s c}
\end{array}\right]=\left[L_{s}\right]\left[\begin{array}{l}
i_{s a} \\
i_{s b} \\
i_{s c}
\end{array}\right]+\left[M_{S R}\right]\left[\begin{array}{l}
i_{r a} \\
i_{r b} \\
i_{r c}
\end{array}\right]} \\
{\left[\begin{array}{l}
\varphi_{r a} \\
\varphi_{r b} \\
\varphi_{r c}
\end{array}\right]=\left[M_{R S}\right]\left[\begin{array}{l}
i_{s a} \\
i_{s b} \\
i_{s c}
\end{array}\right]+\left[L_{R}\right]\left[\begin{array}{l}
i_{r a} \\
i_{r b} \\
i_{r c}
\end{array}\right]}
\end{gathered}
$$

Afin de pouvoir rendre compte des interactions entre les phases, nous avons exprimé chaque matrice résistance et inductance en fonction du nombre de spires des enroulements de la machine. Ce modèle permet en particulier de crées des défauts asymétriques que ce soit au stator ou au rotor par la variation du nombre de spires de l'enroulement de la phase en défaut. Le défaut peut être localisé sur une ou plusieurs phases.

$$
\begin{aligned}
& {\left[R_{S}\right]=\left[\begin{array}{ccc}
R_{s a} & 0 & 0 \\
0 & R_{s b} & 0 \\
0 & 0 & R_{s c}
\end{array}\right]=R_{s}\left[\begin{array}{ccc}
n_{s a} & 0 & 0 \\
0 & n_{s b} & 0 \\
0 & 0 & n_{s c}
\end{array}\right]} \\
& {\left[R_{R}\right]=\left[\begin{array}{ccc}
R_{r a} & 0 & 0 \\
0 & R_{r b} & 0 \\
0 & 0 & R_{r c}
\end{array}\right]=R_{r}\left[\begin{array}{ccc}
n_{r a} & 0 & 0 \\
0 & n_{r b} & 0 \\
0 & 0 & n_{r c}
\end{array}\right]}
\end{aligned}
$$

$n_{s a}$ correspond au nombre de spire de la phase a du stator.

$$
\begin{aligned}
& {\left[L_{S}\right]=\left[\begin{array}{ccc}
L_{s a} & M_{s a b} & M_{s c a} \\
M_{s a b} & L_{s b} & M_{s b c} \\
M_{s c a} & M_{s b c} & L_{s c}
\end{array}\right]} \\
& =\left[\begin{array}{ccc}
n_{s a}^{2} l_{s} & n_{s a} n_{s b} m_{s} & n_{s a} n_{s c} m_{s} \\
n_{s a} n_{s b} m_{s} & n_{s b}^{2} l_{s} & n_{s b} n_{s c} m_{s} \\
n_{s a} n_{s c} m_{s} & n_{s b} n_{s c} m_{s} & n_{s c}^{2} l_{s}
\end{array}\right] \\
& {\left[L_{R}\right]=\left[\begin{array}{ccc}
L_{r a} & M_{r a b} & M_{r c a} \\
M_{r a b} & L_{r b} & M_{r b c} \\
M_{r c a} & M_{r b c} & L_{r c}
\end{array}\right]} \\
& =\left[\begin{array}{ccc}
n_{r a}^{2} l_{r} & n_{r a} n_{r b} m_{r} & n_{r a} n_{r c} m_{r} \\
n_{r a} n_{r b} m_{r} & n_{r b}^{2} l_{r} & n_{r b} n_{r c} m_{r} \\
n_{r a} n_{r c} m_{r} & n_{r b} n_{r c} m_{r} & n_{r c}^{2} l r
\end{array}\right] \\
& {\left[M_{S R}(\theta)\right]=\left[\left(M_{R S}(\theta)\right]^{\mathrm{T}}\right.}
\end{aligned}
$$

\section{B. Choix de la variable à étudier}

L'objectif de cette approche consiste à déterminer les signaux accessibles les plus significatifs pour la détection de défauts. Pour cela, il est nécessaire de tenir compte d'une part de la bande passante du système bouclé et d'autre part du contenu spectral du défaut à surveiller.

\section{De l'influence des boucles de controle}

Il ne faut pas perdre de vue que le système comporte des boucles de contrôle des courants (pour assurer le contrôle vectoriel) et de la vitesse [1]. Ces correcteurs ont pour fonction d'assurer la conformité entre la consigne (ref) et la mesure (y) en agissent sur la grandeur de commande (u).Intuitivement, il est évident que la commande cherchera à compenser toute variation de la grandeur mesurées disponible pour la détection des défauts (les deux courants mesurés et la vitesse mesurées, le vecteur de tension de commande en sortie du dispositif de contrôle vectoriel et la référence de couple en sortie de l'asservissement de vitesse). Considérons le schéma bloc suivant représentant de manière simplifiée la partie contrôle du couple d'un entrainement électrique similaire à notre application.

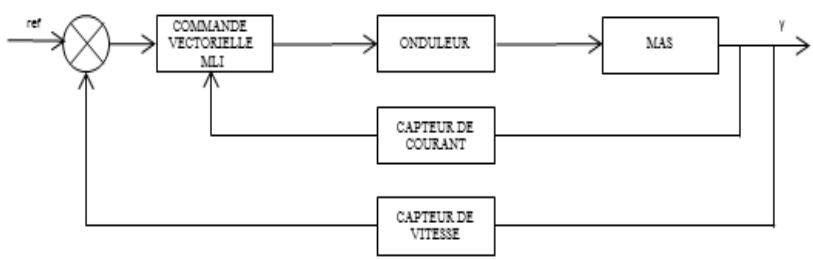

Fig.1. Schéma bloc du système simplifie

\section{Les signatures spéctrales du courant statorique}

Le but de la caractérisation fréquentielle des défauts consiste, en fonction du défaut considéré, à déterminer la (ou les) fréquences (s) liées (s) au défaut et à discriminer la variable (mesurée ou calculée) la plus pertinente dans laquelle cette fréquence peut être isolée. La figure 2 montre la fréquence électrique $\mathrm{f}_{\mathrm{e}}=100 \mathrm{~Hz}$ du spectre du courant statorique (Isa) de la phase a de la machine asynchrone sans court-circuit.

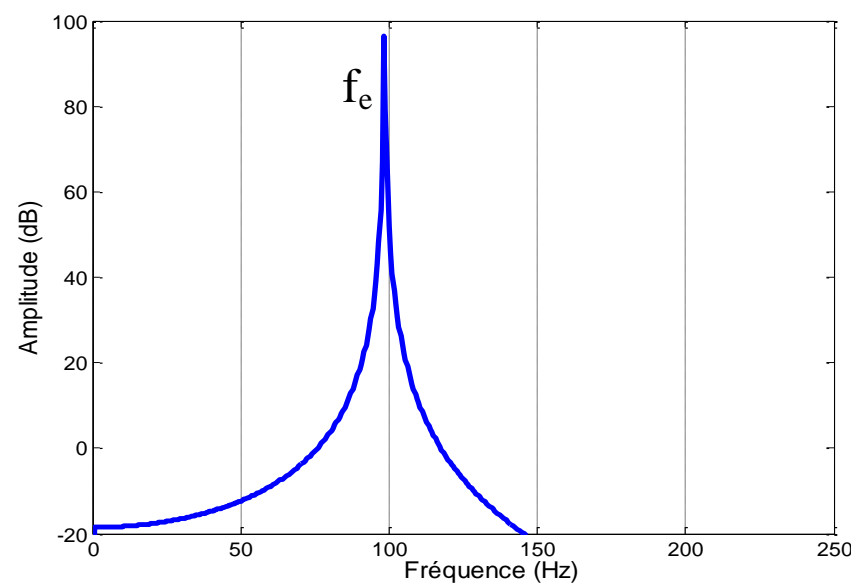

Fig.2. Spectre du courant statorique de la phase a.

La figure 3 montre le spectre du courant statorique (Isa) de la machine asynchrone avec court-circuit sur une phase, on note l'apparition de la fréquence du défaut $3 \mathrm{f}_{\mathrm{e}}(300 \mathrm{~Hz})$. 




Fig.3. Spectre du courant statorique avec défaut sur une phase au stator.

La figure 4 présente les fréquences qui caractérisent le défaut par l'apparition de deux fréquences au tour de la fréquence électrique $(1 \pm 2 \mathrm{~g})$ ou $\mathrm{g}$ représente le glissement.Remarquons que ce défaut sera tré difficile à détecter dans le spectre du courant car l'amplitude de la raie à la fréquence fondamentale est très importante par rapport à celle de la raie recherchée.L'amplitude de défaut considéré consiste en une suppression de $30 \%$ du bobinage d'une phase au rotor ou stator.

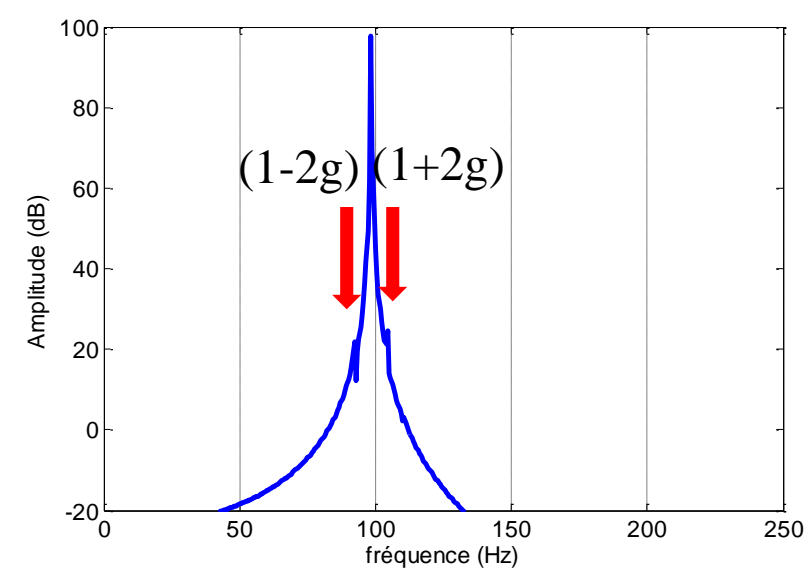

Fig.4. Spectre du courant statorique avec défaut sur une phase au rotor.

Après avoir souligné le choix de la variable a consideré pour détecteter la signature fréquentielle carectéristique du défaut statorique et rotorique, nous avons présenté les caractéristiques fréquentielles des défauts concernant onduleur, capteur de courant et capteur de vitesse.La figure 5 illustre le spectre du courant statorique (Isa) de la machine saine avec défaut d'onduleur (composant ouvert) et la machine saine,on notant l'apparition des raies autour de la fréquence électrique $\mathrm{f}_{\mathrm{e}}$ et autours des fréquences de défauts $\mathrm{kf}_{\mathrm{e}}$ avec( $\left.\mathrm{k}=1,2,3, \ldots \ldots\right)$.

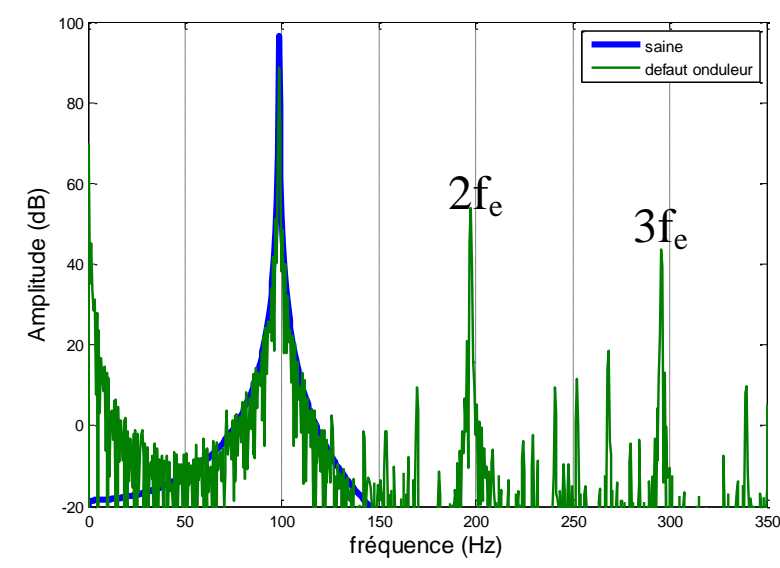

Fig.5. Spectre du courant statorique de la machine saine avec défaut onduleur et la machine saine.

Le défaut capteur de courant considéré consiste en un offset sur la grandeur mesuré (50A soit environ $15 \%$ du calibre).La figure 3 présente le spectre du courant statorique (Isa) de la phase a de la machine lorsque le capteur est défaillant, en retrouve la fréquence de défaut $2 \mathrm{f}_{\mathrm{e}}(200 \mathrm{~Hz})$.

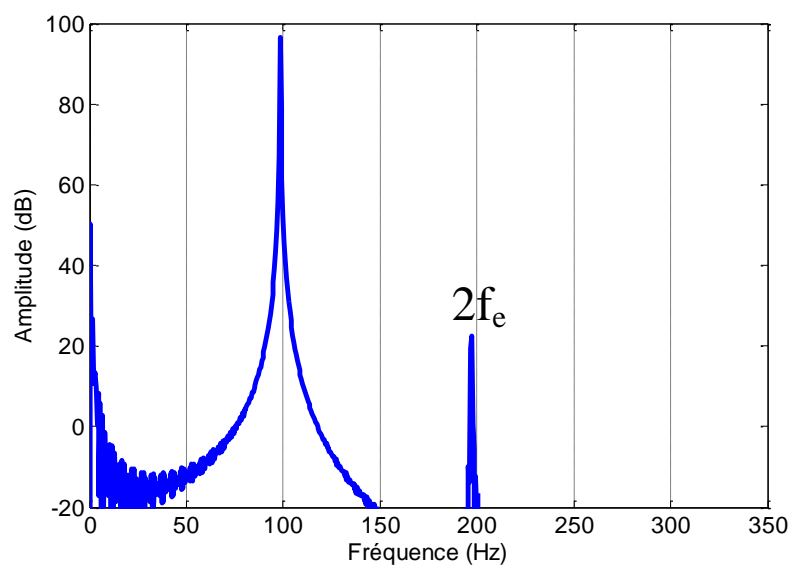

Fig.6.Spectre du courant de la phase a de la machine avec défaut sur le capteur de courant.

En tenant compte de la plage en basse fréquence, on remarque que la fréquence de défaut sur le capteur de vitesse est inexistante (les variables concernées sont continues). La figure 7 présente l'allure de la vitesse mesurée pour une erreur du capteur de $5 \mathrm{Rad} / \mathrm{s}$ (soit 2,5\% de la valeur de référence).On remarque que le contrôle de la vitesse réagit de manière à compenser l'offset qui apparait sur la vitesse mesurée. 




Fig.7. Vitesse mesurée lors d'un défaut capteur de vitesse.

\section{LES EFFETS MECANIQUES DES DEFAUTS DE ROULEMENTS}

Afin de pouvoir utiliser un modèle assez développé de la partie mécanique de l'entrainement électrique, nous avons modélisé une transmission constituée par un arbre flexible avec réducteur. La partie mécanique contient aussi des roulements qui assurent le parfait alignement des axes des pièces tournantes. Nous avons considéré qu'ils n'intervenaient pas dans les équations mécaniques du système lorsqu'ils sont sains. En revanche, lorsqu'un défaut se produit sur ces éléments, nous les prenons en compte par la génération d'un couple de perturbation.

\section{A. Modèle de la transmission}

Le couple moteur est transmis à la roue par le biais d'un réducteur à double train épicycloïdal. Nous ne disposions pas de modelés mécanique de cette transmission. Nous avons donc développé un modèle simple permettant de présenter les deux phénomènes qui nous semblaient les plus importants à considérer : l'élasticité de la transmission et l'irrégularité du couple transmis due aux dents de l'engrènement.la figure 8 présente le modèle $[2,9]$.



Fig.8. Modèle d'une transmission flexible

Les équations de cette transmission sont les suivantes :

$$
\begin{gathered}
C_{1}=J_{1} \frac{\mathrm{d} \omega_{1}}{\mathrm{dt}}+f_{1} \omega_{1}+\frac{C_{1}}{n} \\
C_{1}=J_{2} \frac{\mathrm{d} \omega_{2}}{\mathrm{dt}}+f_{2} \omega_{2}+c_{2} \\
C_{1}=k\left(\frac{\theta_{1}}{n}-\Theta_{2}\right)+d\left(\frac{\omega_{1}}{n}-\omega_{2}\right)
\end{gathered}
$$

\section{B. Prise en compte de l'engrenage}

De nombreuse études ont modélisé l'engrenage par le biais de décomposition en séries de fourier et ont surtout analysé son influence sur les vibrations et les torsions d'arbre. Pour notre étude, nous avons préféré une approche plus intuitive afin d'expliquer les phénomènes physiques entrant en jeu. La validité des hypothèses et de la modélisation est justifiée a posteriori en comparant l'effet du modèle d'engrenage introduit sur la vitesse de rotation de l'axe de la machine sur des relevés issus d'autres travaux [7]. A partir de l'équation, nous pouvons remarquer que l'engrenage intervient au niveau $\mathrm{du}$ couple transmis $(\mathrm{Ct})$.la forme de ce couple en fonction de la position est approchée par les modèles proposés à la figure. Les deux motifs présentés pour le couple transmis permettent de rendre compte des chocs entre les dents. Le premier motif, en pointillés, traduit les chocs entre chaque dent pour la transmission du couple entre les deux parties de l'engrenage. Le deuxième motif, en traits pleins, « représente » le frottement des dents en contact de chaque roue de l'engrenage. Nous avons choisi ce modèle pour la suite des simulations : il traduit une sorte de coefficient de torsion variable en fonction du nombre de dents en contact.

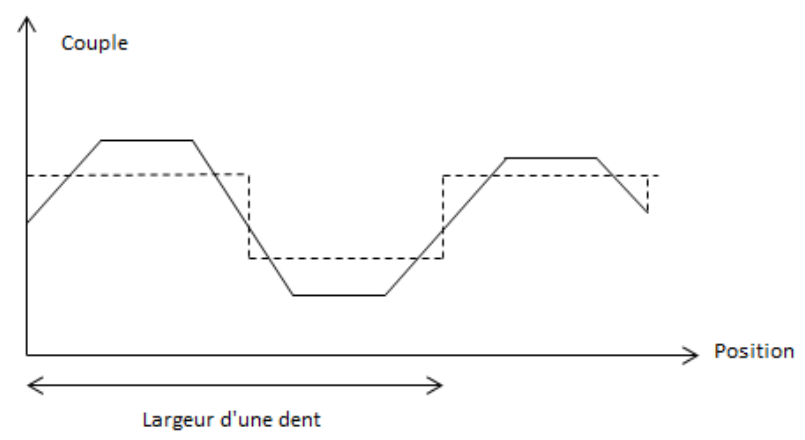

Fig.9. Effet sur le couple transmis par l'engrenage

\section{Les roulements}

Une des raisons les plus fréquentes de défauts dans les machines électriques est liée aux défauts dans les roulements. Nous avons considéré qu'en fonctionnement sain les roulements ne généraient aucun signal. Un défaut sur un roulement entraine une modification de l'alignement de l'arbre de la machine avec l'axe de la machine. Il conduit donc à une modification de la répartition de l'induction au sein de la machine. Cependant, devant la complexité à rendre ces effets, nous avons décidé de ne représenter que le couple perturbateur généré sur l'axe de la machine $[3,6]$.

On utilise la formule suivante :

$$
\mathrm{T}(\alpha)=\sum_{\mathrm{m}=1}^{\mathrm{M}} T_{\mathrm{m}} \sin \left(\alpha k_{m}\right)
$$


Où $\alpha$ est la position de l'axe.

Tm, amplitude du défaut, est choisie proportionnelle au couple appliqué sur l'arbre.

$\mathrm{K}_{\mathrm{m}}$ est donné par $\mathrm{k}_{\mathrm{m}}=m \frac{f_{d}}{f_{r m}}$ où $f_{r m}$ est la vitesse mécanique du rotor et est $f_{d}$ la fréquence correspondant au défaut choisi.

$f_{d}$ est une fonction des dimensions du roulement et devient :

Pour un défaut sur une bille :

$$
f_{b}=\frac{P D}{B D} f_{r m}\left[1-\left(\frac{B D}{P D} \cos \beta\right)^{2}\right]
$$

Avec $\mathrm{BD}=$ diamètre de la bille

$$
\mathrm{PD}=\text { profondeur du roulement }
$$

$\beta=$ angle de contact de la bille sur la couronne interne

-Pour un défaut sur la couronne interne ou externe du roulement :

$$
\left\{\begin{array}{l}
\mathrm{f}_{0}=\frac{\mathrm{n}}{2} f_{r m}\left[1-\frac{B D}{P D} \cos \beta\right] \\
\mathrm{f}_{\mathrm{i}}=\frac{\mathrm{n}}{2} f_{r m}\left[1+\frac{B D}{P D} \cos \beta\right]
\end{array}\right.
$$

Où $\mathrm{n}$ est le nombre de billes du roulement

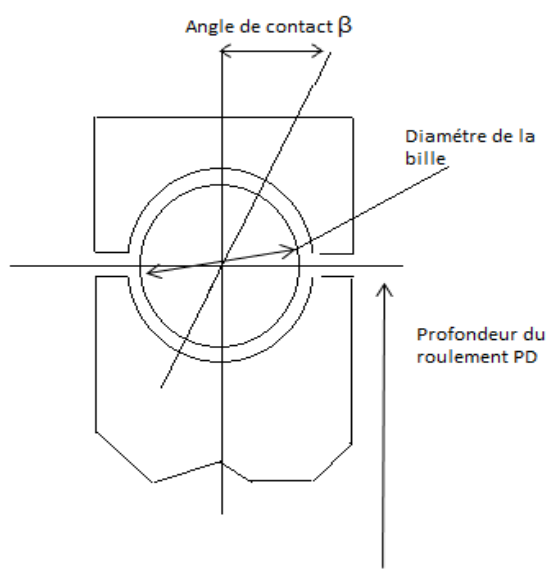

Fig.10.Grandeurs caractéristiques d'un roulement

Nous présentons ici les résultats de simulations effectuées avec les modèles de roulements défaillants introduits. Les simulations ont été effectuées pour une vitesse de $150 \mathrm{rad} / \mathrm{s}$ (soit une fréquence mécanique de $23.9 \mathrm{~Hz}$ ). Les figures suivantes présentent les spectres de la référence de couple (sortie du correcteur de vitesse) pour les différents défauts roulement considérés.

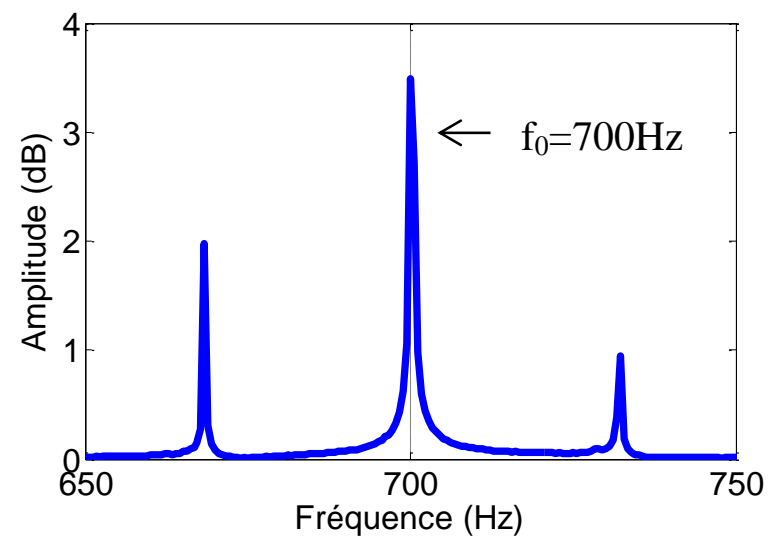

Fig.11. Spectre de la référence de couple pour un défaut sur la bague externe.

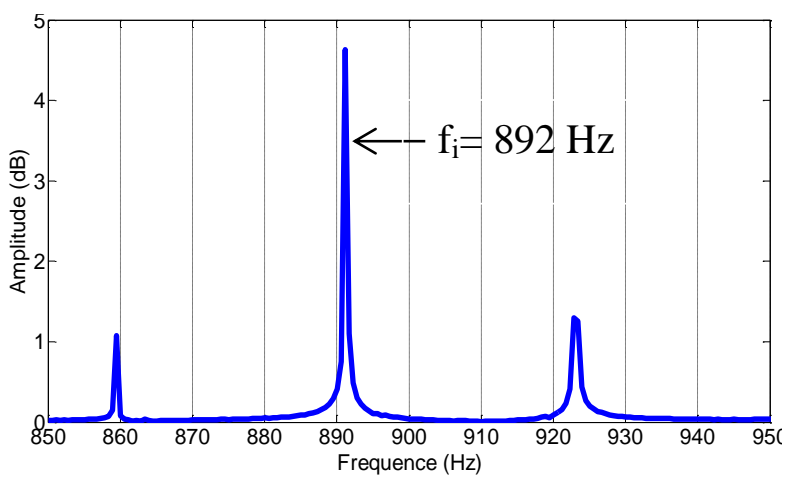

Fig.12. Spectre de la référence de couple pour un défaut sur la bague interne.

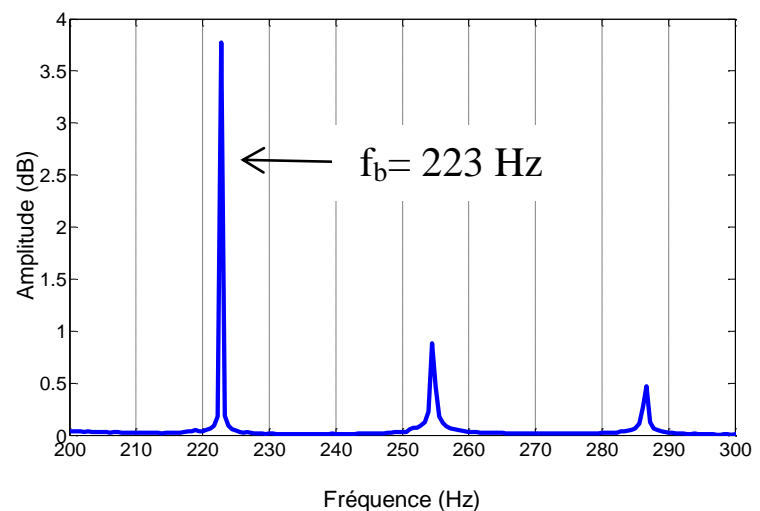

Fig.13. Spectre de la référence de couple pour un défaut sur la bille.

\section{CONCLUSION:}

Apres avoir souligné l'influence du contrôle sur le choix de la variable à considérer pour détecter la signature fréquentielle 
caractéristique du défaut, nous avons présenté les caractéristiques fréquentielles des défauts concernant notre application. Ces fréquences peuvent être variables (fonction de la fréquence électrique ou de la fréquence de rotation mécanique) ou fixe (liées à la MLI). Elles sont résumées dans les tableaux suivants.

A partir du courant de phase

\begin{tabular}{|c|c|c|c|c|}
\hline \multicolumn{5}{|c|}{ Table 1 : Les caractéristiques des défauts électriques } \\
\hline & Stator & Rotor & Onduleur & Capteur courant \\
\hline $\mathrm{f}_{\mathrm{e}}$ & & & & \\
\hline $2 \mathrm{f}_{\mathrm{e}}$ & & & $\mathrm{X}$ & $\mathrm{X}$ \\
\hline $3 \mathrm{f}_{\mathrm{e}}$ & $\mathrm{X}$ & & $\mathrm{X}$ & \\
\hline
\end{tabular}

A partir de la vitesse mécanique

\begin{tabular}{|l|c|c|c|}
\hline \multicolumn{4}{|c|}{ Table 2: Les caractéristiques des défauts mécaniques } \\
\hline & $\begin{array}{c}\text { Défaut roulement } \\
\text { 1 (bague externe) }\end{array}$ & $\begin{array}{c}\text { Défaut roulement } \\
\text { 2 (bague interne) }\end{array}$ & $\begin{array}{c}\text { Défaut roulement } \\
\text { 3 (bille) }\end{array}$ \\
\hline $\mathrm{f}_{0}$ & $\mathrm{X}$ & & \\
\hline $\mathrm{f}_{\mathrm{i}}$ & & $\mathrm{X}$ & \\
\hline $\mathrm{fb}$ & & & $\mathrm{X}$ \\
\hline
\end{tabular}

Les modéles de machine utilisés pour la caractérisation et la validation des algorithmes de surveillance de l'application sont critiquables bien qu'ils permettent une représentation plus réaliste des situations de défaut que les modéles équilibrés couramment adoptés dans la littérature.Ils permettent de représenter des déséquilibres et l'influence des défauts sur l'ensemble des grandeurs. Une carecterisation de degré de validité du modèle développé est à faire.La difficulté rencontrée avec les méthodes de traitement du signal tient à la mise en évidence des raies dont la fréquence et l'amplitude varient avec le point de fonctionnement.

\section{REFERENCES}

[1] S.Diao, Z.Makni,J-F.Bisson "Sensor Fault Diagnosis for Improving the Availability of Electrical Drives" Industrial Electronics Society, IECON2013-39th Annual Conference of the IEEE.

[2] Trajin, Regnier.J, Faucher.J Bearing "Fault indicator in induction machine using stator current spectral analysis" Power Electronics, machine and drives, 2008. PEMD. 4th IET Conférence On

[3] Xu Boqiang, Zhou Huihuan,Sun Liling “ weak-signal detection and the application in detection of electrical motors faults" Electrical Machines and Systems. ICEMS, International Conference On, 2007.

[4] Andrian Ceban " Méthode globale de diagnostic des machines électriques" Thèse de doctorat, université Lille de nord de France, 2012.

[5] Mohamed el kamel.O “ Surveillance et diagnostic des défauts rotoriques et mécaniques de la machine asynchrone avec alimentation équilibrée ou déséquilibrée" Thèse de doctorat, université de lorraine, 2012.

[6] Sanna Jelassi “ Impact d'un court-circuit interne au stator d'une machine asynchrone sur les phénomènes générés par la denture" Thèse de doctorat, Université d'Artois et de L'université de Monastir, 2011.

[7] Andrian Ceban " Méthode globale de diagnostic des machines électriques" Thèse de doctorat, université Lille de nord de France, 2012.

[8] Vincent Devanneaux “ Modélisation des machines asynchrone triphasées à cage d'écureuil en vue de la surveillance et du diagnostic" Thèse de doctorat, Institut National Polytechnique de Toulouse, 2002.

[9] Bertrand Raison “ Détection et localisation de défaillance sur un entraiinement électrique " Thése de doctorat , Institut National Polytechnique de Grenoble, 2000. 